\title{
Efectos de espaciamientos sobre el crecimiento y rendimiento del fríjol caupí cultivar Caupicor 50
}

\section{Effects of spacings on the growth and yield of cowpea beans cultivar Caupicor 50}

Carlos E. Cardona-Ayala ${ }^{1 *}$; Félix Pastrana-Atencia ${ }^{2}$; Hermes Araméndiz-Tatis ${ }^{3}$ Miguel Espitia-Camacho ${ }^{4}$; Carlos E. Cardona-Villadiego ${ }^{5}$

11.ng. Agrónomo, Ph.D. Universidad de Córdoba, Facultad de Ciencias Agrícolas. Montería - Córdoba, Colombia; email: cecardona@correo.unicordoba.edu.co; iD https://orcid.org/0000-0002-9607-3858

${ }^{2}$ Ing. Agrícola, M.Sc. Universidad de Sucre - Sincelejo, Colombia; email: felixpastra22@gmail.com; (iD) https://orcid.org/0000-0003-3302-8440

${ }^{3}$ Ing. Agrónomo, Ph.D. Universidad de Córdoba, Facultad de Ciencias Agrícolas. Montería - Córdoba, Colombia; email: haramendiz@correo.unicordoba.edu.co; (iD) https//orcid.org/0000-0002-2585-6273

${ }^{4}$ Ing. Agrónomo, Ph.D. Universidad de Córdoba, Facultad de Ciencias Agrícolas. Montería - Córdoba, Colombia; email: mmespitia@correo.unicordoba.edu.co; (iD https://orcid.org/0000-0001-7382-9643

${ }^{5}$ Ing. Agrónomo, M.Sc., Investigador particular. Montería - Córdoba, Colombia; email: carloscardona2993@gmail.com; (iD https://orcid.org/0000-0002-6466-6923 *autor para correspondencia: cecardona@correo.unicordoba.edu.co

Cómo citar: Cardona-Ayala, C.E.; Pastrana-Atencia, F.; Araméndiz-Tatis, H.; Espitia-Camacho, M.; Cardona-Villadiego, C.E. 2021. Efectos de espaciamientos sobre el crecimiento y rendimiento del fríjol caupí Caupicor 50 Rev. U.D.C.A Act. \& Div. Cient. 24(2):e2139. http://doi. org/10.31910/rudca.v24.n2.2021.2139

Artículo de acceso abierto publicado por Revista U.D.C.A Actualidad \& Divulgación Científica, bajo una Licencia Creative Commons CC BY-NC 4.0

Publicación oficial de la Universidad de Ciencias Aplicadas y Ambientales U.D.C.A, Institución de Educación Superior Acreditada de Alta Calidad por el Ministerio de Educación Nacional.

Recibido: junio 19 de 2020

Aceptado: noviembre 3 de 2021

Editado por: Helber Adrián Arévalo Maldonado

\section{RESUMEN}

El fríjol caupí es una leguminosa importante en la región Caribe de Colombia, por su contribución a la dieta de sus pobladores y por ser una alternativa de ingresos para pequeños productores; sin embargo, los rendimientos obtenidos no superan los $600 \mathrm{~kg} \mathrm{ha}^{-1}$, debido al desconocimiento del efecto de las prácticas culturales en la especie, como en los diversos cultivares. El objetivo del estudio fue evaluar el efecto de diferentes espaciamientos e hidrogel sobre características vegetativas y reproductivas del fríjol caupí cultivar Caupicor 50, en el departamento de Sucre, del Caribe colombiano. Se utilizó el diseño experimental parcelas sub-subdivididas, con tres repeticiones (bloques). Se combinaron dos distancias entre hileras: 60 y $80 \mathrm{~cm}$ en la parcela principal, tres distancias entre plantas: 20 , 30 y $40 \mathrm{~cm}$ en la subparcela e hidrogel: 0 y $5 \mathrm{~g} /$ planta en la sub-subparcela. Las variables evaluadas fueron: altura de planta, área foliar, índice de área foliar, tasa de crecimiento del cultivo, número de vainas/planta, longitud de vaina, número de semillas/ vaina, peso de cien semillas y rendimiento de semillas/ha. El mayor rendimiento, se estimó con $20 \mathrm{~cm}$ entre plantas y $60 \mathrm{~cm}$ entre hileras (83.333 plantas ha ${ }^{-1}$ ), con un rendimiento de $2.027 \mathrm{~kg}$ ha-1, asociado a mayor área foliar, índice de área foliar y tasa de crecimiento del cultivo y, a menor número de vainas por planta, longitud de vaina y peso de cien semillas.

Palabras clave: Competencia entre plantas; Densidad de plantas; Seguridad alimentaria; Sequía; Vigna unguiculata. 


\section{ABSTRACT}

Cowpea bean is an important legume in the Caribbean region of Colombia, for its contribution to the diet of its habitants and being an alternative income for small producers. Nevertheless, the yields obtained do not exceed $600 \mathrm{~kg} \mathrm{ha}^{-1}$, due to the unknown of the effect of cultural methods on the species, as in the different cultivars. The purpose of the study was to evaluate the effect of different spacing and hydrogel on vegetative and reproductive characteristics of cowpea beans, cultivar Caupicor 50 in the department of Sucre of the Caribbean region of Colombia. A split-plot design was used, with three replications (blocks). Two distances between rows: 60 and $80 \mathrm{~cm}$ in the main plot, three spaces between plants: 20, 30, and $40 \mathrm{~cm}$ in the subplot, and hydrogel: 0 and $5 \mathrm{~g} /$ plant in the sub-sub plot were combined. The variables evaluated were: plant height, leaf area, leaf area index, crop growth rate, number of pods per plant, pod length, number of seeds per pod, the weight of one hundred seed, and seed yield/ha. The highest yield, 2,027 kg ha', was estimated with $20 \mathrm{~cm}$ between plants and $60 \mathrm{~cm}$ between rows $\left(83,333\right.$ plants $\left.\mathrm{ha}^{-1}\right)$ and was associated with greater leaf area, leaf area index and crop growth rate, and a lower number of pods per plant, pod length and weight of one hundred seeds.

Keywords: Vigna unguiculata; Food safety; Drought; Plant density; Competition between plants.

\section{INTRODUCCIÓN}

El fríjol caupí (Vigna unguiculata L. Walp) es una leguminosa adaptada a las zonas semiáridas tropicales y subtropicales (Huynh et al. 2013), cultivada en suelos de sabanas, con cultivares tradicionales de baja capacidad productiva, inadecuados sistemas de siembra y sin insumos agrícolas (Kamara et al. 2018). Esta especie, se destaca por su contribución a la seguridad alimentaria y nutricional en las poblaciones vulnerables, de países en vía de desarrollo, ya que posee alto contenido de proteína y micronutrientes, como hierro y zinc (Kuruma et al. 2019) y bajos costos de producción (Costa Junior et al. 2017), pero sus rendimientos no superan los $600 \mathrm{~kg} \mathrm{ha}^{-1}$.

En la agricultura, el déficit de agua en el suelo afecta, principalmente, las áreas tropicales, por ser las lluvias deficientes, en algunos casos y su distribución errática, circunstancia que incide en el desarrollo de la planta, generando modificaciones anatómicas, morfológicas, fisiológicas y bioquímicas, causando pérdidas directamente relacionadas con su duración, severidad y etapa de desarrollo del cultivo (Alves Bastos et al. 2011). En el fríjol caupí, la sequía, afecta varias características, como el diámetro del tallo, el área foliar, el número de hojas, el número de vainas por plantas, el peso de vainas, el peso de semillas y el rendimiento (De Sousa Monteiro et al. 2017).

Los cultivares modernos ameritan tecnologías que permitan expresar su potencial de rendimiento, requieren de la cantidad de agua adecuada y densidad de población óptima, que son determinantes en el manejo agronómico del cultivo. En este sentido, la densidad de plantas tiene como objetivo aumentar la eficiencia del dosel en interceptar la radiación. De Sousa Mendes et al. (2005) reportaron aumentos que en el porcentaje de luz interceptada e índice de área foliar de caupí entre 50 y 206,5\%, respectivamente, cuando la densidad de plantas se incrementó de 41.666 a 166.666 plantas ha ${ }^{-1}$.

El uso de polímeros hidrófilos, conocidos como hidrogeles, es una alternativa que se usa en cultivos perennes leñosos y en cultivos agrícolas, para contrarrestar el déficit hídrico en los suelos (Souza Lopes et al. 2017), puesto que pueden aumentar el contenido y la disponibilidad de agua para las plantas (Pedroza-Sandoval et al. 2015). En este sentido, el fríjol caupí, a pesar de ser tolerante a la sequía, los prolongados períodos sin precipitaciones afectan los rendimientos, especialmente, por la disminución del número de vainas por aborto de flores. El uso de hidrogeles se viene incrementando, porque facilita la retención de agua y porque permite incrementar la producción; por ejemplo, Souza Pereira et al. (2018) reportaron un aumento en la producción de granos de frijol caupi hasta del 46,05\%, con el uso del hidrogel.

El mejoramiento de los rendimientos es deseable en cualquier sistema de producción y para lograrlo hay que desarrollar tecnologías que contribuyan a tal fin. En Colombia son pocos los estudios sobre densidad de población y uso de hidrogeles, como tecnología para mitigar los efectos negativos de la sequía en fríjol caupí y, en especial, en los diferentes cultivares. Por tanto, el objetivo del estudio fue evaluar el efecto de diferentes espaciamientos e hidrogel sobre las características vegetativas y reproductivas del fríjol caupí cultivar Caupicor 50, en el departamento de Sucre, del Caribe colombiano.

\section{MATERIALES Y MÉTODOS}

Localización. El estudio, se realizó entre febrero a mayo de 2017, en el corregimiento de La Arena, municipio de Sincelejo, departamento de Sucre, Colombia, situado a $9^{\circ} 18>$ de latitud Norte y $75^{\circ} 23$ > de longitud Oeste; con clima tropical seco o de sabana (Aw), según la clasificación climática de Köppen; con temperatura media anual de $27,15^{\circ} \mathrm{C}$; altura promedio de $213 \mathrm{~m}$ s.n.m.; humedad relativa media anual del $80 \%$ y precipitación media anual de 1.192 mm (IDEAM, 2014).

Condiciones edafoclimatológicas. Durante el experimento, se registraron $324 \mathrm{~mm}$ de precipitación y una temperatura media de $29{ }^{\circ} \mathrm{C}$. El suelo, donde se realizó el experimento, corresponde a un vertisol, con $\mathrm{pH} 7,74,2,87$ \%, de materia orgánica y una CIC, de $77,2 \mathrm{cmol} \mathrm{kg}^{-1}$.

Material vegetal y manejo agronómico. Se utilizó la variedad comercial Caupicor 50, cultivar desarrollado por la Universidad de Córdoba, de hábito semipostrado, ciclo de 60 días y tolerante a sequía. Asimismo, semilla de excelente calidad fitosanitaria, pureza genética, calidad física y porcentaje de germinación superior al $95 \%$. El manejo agronómico, se realizó de acuerdo con las recomendaciones de la cartilla Manejo Agronómico del fríjol caupí en el Caribe colombiano (Araméndiz-Tatis et al. 2019). 
Diseño experimental. Se utilizó el diseño parcelas subdivididas con tres repeticiones constituidas por bloques. Se evaluaron dos distancias entre hileras: 60 y $80 \mathrm{~cm}$, en las parcelas principales, tres distancias entre plantas: 20, 30 y $40 \mathrm{~cm}$ en las subparcelas e hidrogel biodegradable al suelo: 0 y $5 \mathrm{~g} /$ planta en las sub-subparcelas, para un arreglo factorial $2 \times 3 \times 2$. Las combinaciones entre los niveles de los factores distancia entre hileras (DH) y entre plantas (DP) equivalen a densidades de plantación de 83.333, 55.555, 41.666, $62.500,41.666$ y 31.250 plantas $\mathrm{ha}^{-1}$, respectivamente. Las parcelas principales, se trazaron con las dimensiones de $6 \times 8 \mathrm{~m}$, para un área de $48,0 \mathrm{~m}^{2}$. El factor hidrogel biodegradable $(\mathrm{H})$ utilizado, también conocido como hidrorretenedor (Souza Pereira et al. 2018), es derivado de almidón de yuca y fue producido en el laboratorio de Aguas y suelos Agrícolas de la Universidad del Valle; se aplicó alrededor de cada plántula, en un radio de $15 \mathrm{~cm}$ e incorporado a 10 $\mathrm{cm}$ de profundidad, tres días después de la siembra, coincidiendo con el inicio de la emergencia de las plántulas.

Caracterización del crecimiento y la Producción. Las variables de respuesta fueron descritas, de acuerdo con los trabajos de Souza Pereira et al. (2018) y Soares de Melo et al. (2018).

Fase vegetativa. Las variables de esta fase fueron medidas a los 35 días después de la emergencia, coincidiendo con la plena floración y comienzo de formación de vainas y sus semillas. Altura de planta (AP): medida desde el cuello de la raíz hasta el último nudo del tallo principal de tres plantas de los surcos centrales en plena competencia. Área foliar (AF): mediante muestreos destructivos en tres plantas, con el método de relación peso/área; se expresó en $\mathrm{cm}^{2}$. Índice de área foliar (IAF): se utilizó la relación entre el área foliar y el área de suelo sobre las tres plantas de las líneas centrales de cada unidad experimental. Tasa de crecimiento del cultivo (TCC): ganancia de biomasa en el área ocupada por la planta, a partir del promedio de tres plantas por cada unidad experimental. Las muestras, se llevaron a estufa de secado a $70{ }^{\circ} \mathrm{C}$, hasta alcanzar peso constante.
Fase reproductiva. Las variables de esta fase, se midieron a los 65 días al momento de la cosecha. Longitud de la vaina (LV): longitud promedio de 10 vainas maduras, tomadas al azar, de la población de plantas de cada parcela, en $\mathrm{cm}$. Número de vainas por planta (NVP): promedio de cinco plantas, tomadas al azar, en competencia, en cada parcela. Número de semillas por vaina (NSV): número promedio de semillas de 10 vainas maduras, tomadas de cinco plantas, seleccionadas al azar, en competencia, en cada parcela. Peso de cien semillas (PCS): peso de 100 semillas, en gramos, seleccionadas al azar, en 20 vainas, de cada unidad experimental. Rendimiento de semillas (REND): peso de las semillas de la parcela útil expresado en $\mathrm{kg} \mathrm{ha}^{-1}$, con humedad ajustada a $14 \%$.

Análisis de datos. Para AP, AF, IAF y TCC, se realizaron análisis varianza, de acuerdo con el modelo lineal aditivo del diseño en parcelas subdivididas; se verificó el cumplimiento de los supuestos de normalidad, de homogeneidad de varianzas, de aditividad e independencia de los errores. Las pruebas de comparación de medias, se hicieron para las variables que resultaron significativas. Adicionalmente, se estimaron modelos de regresión para las variables de la fase reproductiva, que resaltaron significativas en el análisis de varianza: LV, NVP, PCS y REND, cumpliendo los supuestos de la estadística paramétrica e, igualmente, análisis de correlación para todas las variables. Los análisis estadísticos, se realizaron con el software SAS versión 9.1.

\section{RESULTADOS Y DISCUSIÓN}

Características de crecimiento. El análisis de varianza para las interacciones de primer y segundo orden (Tabla 1), registró ausencia de significancia ( $\mathrm{p} \geq 0,05$ ), lo que indica que los efectos de los factores estudiados sobre las variables de respuesta son independientes, por lo cual, tienen importancia los efectos principales de cada factor estudiado, que se evalúan mediante pruebas de comparación de medias, como lo sostienen Da Camara et al. (2018) y concuerda con los estudios de De Carvalho Bezerra et al. (2009) y Barrera et al. (2021).

Tabla 1. Cuadrados medios del análisis de varianza para las variables altura de la planta (AP), área foliar (AF), índice de área foliar (IAF) y tasa de crecimiento del cultivo (TCC), a los 35 días después de la emergencia del fríjol caupí cultivar Caupicor 50, a diferentes espaciamientos.

\begin{tabular}{|c|c|c|c|c|}
\hline FV & AP & AF & IAF & TCC \\
\hline DH & 15,38 & 1055718,58 & $10,95^{*}$ & $29,36^{*}$ \\
\hline Error a & 3,69 & 1152109.74 & 0,45 & 0,58 \\
\hline DP & $798,41^{*}$ & $46844993,14^{* *}$ & $4,85^{* *}$ & $22,07^{* *}$ \\
\hline DP x DH & 193,32 & 283216,54 & 0,24 & 2,13 \\
\hline Error b & 122,37 & 346447,39 & 0,12 & 0,71 \\
\hline HG & 1,36 & 156173,82 & 0,02 & 0,41 \\
\hline DH x HG & 18,39 & 90087,02 & 0,02 & 0,33 \\
\hline DP x HG & 46,18 & 61426,65 & 0,01 & 0,33 \\
\hline DH x DP x HG & 14,77 & 23185,12 & 0,01 & 0,34 \\
\hline Error c & 50,69 & 584083,40 & 0,32 & 0,84 \\
\hline Media & 111,38 & 8852,54 & 4,42 & 8,64 \\
\hline CV (\%) & 6,39 & 8,63 & 12,73 & 10,62 \\
\hline
\end{tabular}

DH: Efecto principal del factor distancia entre hileras; DP: Efecto principal del factor distancia entre plantas; HG: Efecto principal del factor Hidrogel; DH x DP, DH x HG; DP x HG y DH x DP x HG: efectos de las interacciones de primer y segundo orden; CV: coeficiente de variación. *efecto significativo al $5 \%$; **efecto significativo al $1 \%$. 
Se estimaron diferencias significativas $(\mathrm{p}<0,05)$ para IAF y TCC, en el factor DH, mientras que para DP, hubo diferencias altamente significativas $(\mathrm{p}<0,01)$, en sus efectos sobre AP, AF, IAF y TCC. El factor hidrogel $(\mathrm{H})$ no influyó en las variables estudiadas, posiblemente, porque durante el desarrollo de la investigación, la precipitación acumulada fue de $340 \mathrm{~mm}$, por encima de la evapotranspiración del cultivo, estimada por Apáez Barrios et al. (2016) y acorde con las necesidades del cultivo, de acuerdo con lo reportado por Alves Bastos et al. (2012). Esta precipitación en la estación seca del año es considerada anormal y es consecuencia del cambio climático, que genera precipitaciones erráticas, en muchas partes del mundo.

Las DH de 60 y $80 \mathrm{~cm}$ no influyeron significativamente en la AP y AF total de la planta, en contraste con el IAF y la TCC, que fueron mayores en la distancia de $60 \mathrm{~cm}$ (Tabla 2), debido, en parte, a que el AF cubre una menor área de suelo. El AF de mayor magnitud, se presentó en el espaciamiento de $40 \mathrm{~cm}$ entre plantas, siendo 22,5 y 36,0\% más alta que en las distancias de 30 y 20 $\mathrm{cm}$, respectivamente. Es evidente que, las plantas al disponer de mayor espacio dentro de la hilera, desarrollaron mayor AF, pero, a su vez, menor IAF, que fue un poco más del $20 \%$ más alto en los distanciamientos de 20 y $30 \mathrm{~cm}$, dentro de la hilera.
La AP está asociada con la producción de nudos, hojas y ramas y alcanza incrementos importantes al iniciar la floración (Hissene et al. 2016), para, posteriormente, desviar los fotoasimilados a las estructuras reproductivas (Gonçalves et al. 2017). En materiales semipostrados, como el del presente estudio, el incremento en AP observado en el tallo principal, continúa en la medida en que la planta sigue floreciendo, para producir más vainas y generar una segunda y tercera cosecha. La mayor AP, se presentó en DP de 40 $\mathrm{cm}$, la cual, fue 6,7 y 13,6 \% más alta que en las distancias de 30 y 20 $\mathrm{cm}$, respectivamente (Tabla 2). La AP presentó correlación positiva con AF, NVP y PCS y negativa, con IAF y TCC (Tabla 5).

El AF es un parámetro importante en la determinación del nivel óptimo, con el cual, se puede maximizar la mayor intercepción de radiación solar y lograr una mayor productividad de un cultivo. El caupí posee dos etapas: una, en la cual, el número de hojas de la planta aumenta a un ritmo más lento y, la otra, con una tasa más alta de producción de hojas por planta, que se relaciona con la aparición de ramas primarias y secundarias. La existencia de diferencias significativas en el AF entre los niveles de DP, de 20, 30 y $40 \mathrm{~cm}$ (Tabla 2), posiblemente, obedezca a diferencias en el filocrono, que podría ser más alto en el mayor distanciamiento entre plantas, es decir, en la densidad de plantas más baja (Hissene

Tabla 2. Valores medios de las variables de crecimiento altura de la planta (AP), área foliar (AF), índice de área foliar (IAF) y tasa de crecimiento del cultivo (TCC) del fríjol caupí cultivar Caupicor 50, a diferentes espaciamientos.

\begin{tabular}{|c|c|c|c|c|c|}
\hline \multirow{2}{*}{\multicolumn{2}{|c|}{ Distancias (m) }} & \multicolumn{4}{|c|}{ Variables de crecimiento } \\
\hline & & \multirow{2}{*}{$\begin{array}{l}\mathbf{A P}(\mathbf{c m}) \\
112,03 \mathrm{a} \\
\end{array}$} & \multirow{2}{*}{$\begin{array}{c}\mathbf{A F}\left(\mathbf{c m}^{2}\right) \\
9023,8 \mathrm{a} \\
\end{array}$} & \multirow{2}{*}{$\begin{array}{c}\text { IAF } \\
4,97 \mathrm{a} \\
\end{array}$} & \multirow{2}{*}{$\begin{array}{c}\begin{array}{c}\text { TCC } \\
\left(\mathrm{g} \mathrm{cm}^{-2} \mathbf{d}^{-1}\right)\end{array} \\
9,54 \mathrm{a}\end{array}$} \\
\hline $\mathrm{DH}$ & 0,60 & & & & \\
\hline DH & 0,80 & $110,73 \mathrm{a}$ & $8681,3 \mathrm{a}$ & $3,87 \mathrm{~b}$ & $7,73 \mathrm{~b}$ \\
\hline \multirow{3}{*}{ DP } & 0,20 & $103,19 \mathrm{~b}$ & $7072,40 \mathrm{c}$ & $5,15 \mathrm{a}$ & $10,20 \mathrm{a}$ \\
\hline & 0,30 & $111,45 \mathrm{ab}$ & $8506,90 \mathrm{~b}$ & $4,11 \mathrm{~b}$ & $7,90 \mathrm{~b}$ \\
\hline & 0,40 & $119,50 \mathrm{a}$ & $10978,40 \mathrm{a}$ & $4,00 \mathrm{~b}$ & $7,81 \mathrm{~b}$ \\
\hline
\end{tabular}

Medias con las mismas letras no difieren significativamente, según la prueba de Tukey al 5 \% de probabilidad. DH: distancia entre hileras; DP: distancia entre plantas.

et al. 2016). Por otra parte, un mayor distanciamiento implica una menor competencia por luz y la posibilidad de formación de mayor número de ramas y hojas. El AF presentó correlación positiva con TCC y negativa, con REND (Tabla 5).

El IAF registró incrementos con la disminución de la DH y DP, es decir, con el aumento de la densidad de población. El mayor IAF, se presentó en DP, de $20 \mathrm{~cm}$ entre plantas y fue 20,2 y 22,3\% más alto que en las distancias de 30 y $40 \mathrm{~cm}$, respectivamente, mientras que para $\mathrm{DH}$, el IAF, en el espaciamiento de $0,60 \mathrm{~m}$, fue un 22,1 \% más alto que en el espaciamiento de $80 \mathrm{~cm}$ (Tabla 2). Resultado similar fue encontrado por Kamara et al. (2018), con cultivares mejorados precoces y tardíos de origen africano, con densidades de 133.333, 266.666 y 400.000 plantas ha ${ }^{-1}$; para estos investigadores existe un IAF óptimo, para capitalizar una alta tasa de fotosíntesis y rendimiento; densidades superiores a la óptima, generan sombreamiento y afectan los componentes del rendimiento, independiente del hábito de crecimiento del cultivar, como consecuencia de la competencia intraespecífica. Asimismo, la afectación del rendimiento puede ser mucho más negativa, si el ciclo concuerda con un déficit hídrico en la floración y llenado de granos (Cardoso et al. 2018). El IAF presentó correlación positiva con TCC y REND y negativa, con AP, NVP y PCS (Tabla 5).

La TCC fue más alta en los menores DH y DP, junto con el IAF (Tabla 2). Esto indica que, en las densidades de plantas más altas, se produjo mayor cantidad de masa seca por unidad de superficie por día, lo cual, concuerda con De Carvalho Bezerra et al. (2017), quienes ajustaron un modelo de TCC en función de cinco densidades de plantas crecientes, desde 100.000 hasta 500.000 plantas ha ${ }^{-1}$. Esta variable presentó correlación positiva con IAF y REND y negativa, con AP, NVP y PCS (Tabla 5).

Características de producción. El análisis de varianza registró diferencias significativas $(p<0,05)$ en el factor $\mathrm{DH}$, para las variables NVP y REND, mientras que, para DP, se estimaron diferencias significativas ( $p<0,05)$, en LV, NVP, PCS y REND (Tabla 3). 
La ausencia de significancia de las interacciones sugiere que el cultivar de fríjol Caupicor 50 responde de manera independiente a DH y DP y el análisis se circunscribe a cada factor de manera independiente. Da Camara et al. (2018) reportaron resultados similares en Brasil, con el cultivar manteiga.

El NSV, se mantuvo constante en DH y DP (Tabla 4), lo cual, indica que esta característica es poco afectada por el ambiente y tiene baja plasticidad fenotípica. Resultados similares fueron obtenidos por
Jakusko et al. (2013) y por El Naim et al. (2010), con los cultivares IT89KD-288, IT89KD391, IT97K-499-35, Buff, Haydoob y Eien Elgazal, respectivamente; no obstante, Wubetu (2018), en fríjol mungo (Vigna radiata L.), encontraron efectos independientes significativos de los dos factores, con mayor NSV, en DH y DP de 25 y $5 \mathrm{~cm}$, respectivamente. Por lo tanto, esta respuesta podría estar más relacionada con la especie o cultivar.

Tabla 3. Cuadrados medios del análisis de varianza para las variables número de semillas por vaina (NSV), longitud de vainas (LV), número de vainas por planta (VP), peso de cien semillas (PCS) y rendimiento de semillas (REND) de frijol caupí cultivar Caupicor 50, a diferentes espaciamientos.

\begin{tabular}{|c|c|c|c|c|c|}
\hline FV & NSV & LV & NVP & PCS & REND \\
\hline DH & 0,001 & 16,40 & $121,40^{*}$ & 4,28 & $335113,63^{*}$ \\
\hline Error a & 2,53 & 3,06 & 4,67 & 0,25 & 10013,21 \\
\hline DP & 2,99 & $25,44^{*}$ & $40,02^{*}$ & $9,88^{* *}$ & $884017,80^{* *}$ \\
\hline DH x DP & 0,09 & 7,68 & 2,11 & 3,39 & 70700,58 \\
\hline Error b & 1,14 & 4,56 & 6,83 & 0,83 & 1858,00 \\
\hline HG & 0,04 & 2,61 & 2,15 & 0,22 & 15297,57 \\
\hline DH x HG & 0,30 & 1,56 & 0,96 & 0,24 & 11289,06 \\
\hline DP x HG & 0,17 & 1,02 & 2,01 & 2,12 & 4258,55 \\
\hline DH x DP x HG & 0,06 & 2,19 & 0,95 & 0,01 & 642,65 \\
\hline Error c & 0,63 & 1,67 & 7,19 & 0,77 & 10949,59 \\
\hline Media & 13,61 & 15,6 & 16,66 & 15,80 & 1719,05 \\
\hline CV (\%) & 5,82 & 8,27 & 16,09 & 5,57 & 6,09 \\
\hline
\end{tabular}

DH: Efecto principal del factor distancia entre hileras; DP: Efecto principal del factor distancia entre plantas; HG: Efecto principal del factor Hidrogel; DH x DP, DH x HG; DP x HG y DH x DP x HG: efectos de las interacciones de primer y segundo orden; CV: coeficiente de variación. *efecto significativo al $5 \%$; **efecto significativo al $1 \%$.

La LV estuvo influenciada por el factor DP, pero fue similar en DH (Tabla 4). La mayor magnitud de esta variable, se expresó en DP de $40 \mathrm{~cm}$, en la que se produjeron vainas más largas, en mayor número y con semillas más pesadas, pero disminuyó el rendimiento de semillas y presentó correlación negativa con la TCC (Tabla 5). La diferencia en LV entre la DP más alta, $40 \mathrm{~cm}$ y la más baja, 20 $\mathrm{cm}$ fue de $17,5 \%$. Resultados previos fueron reportados por De Sousa Monteiro et al. (2017) y Cardoso et al. (2018), atribuido al efecto de la competencia intraespecífica por los fotoasimilados y es válido para cualquier tipo de cultivar de fríjol caupí, independiente de su hábito de crecimiento.
Jiménez-Galindo \& Acosta-Gallegos (2013) indican que la LV es mayor cuando hay mayor espacio entre las plantas, capaces de producir órganos morfológicos de mayor tamaño y peso. Adicionalmente, Makoi et al. (2010) reportaron que, en situaciones de alta densidad de plantas, los minerales, probablemente, se convierten en limitantes en la competencia de planta a planta, ya que las concentraciones de $\mathrm{P}, \mathrm{K}, \mathrm{Ca}, \mathrm{Mg}, \mathrm{S}, \mathrm{Fe}, \mathrm{Cu}, \mathrm{Zn}, \mathrm{Mn}$ y B son más bajas en la rizosfera de plantas establecidas bajo alta densidad.

Tabla 4. Valores medios de las variables de producción número de semillas por vaina (NSV), longitud de vainas (LV), número de vainas por planta (VP), peso de cien semillas (PCS) y rendimiento de semillas (REND) de fríjol caupí cultivar Caupicor 50, a diferentes espaciamientos.

\begin{tabular}{|c|c|c|c|c|c|c|}
\hline \multirow{2}{*}{\multicolumn{2}{|c|}{ DISTANCIAS (m) }} & \multicolumn{4}{|c|}{ VARIABLES DE CRECIMIENTO } & \multirow{2}{*}{$\begin{array}{l}\text { REND } \\
\text { (kg/ha) }\end{array}$} \\
\hline & & NSV & $\mathrm{LV}(\mathrm{cm})$ & NVP & PCS (g) & \\
\hline \multirow{2}{*}{ DH } & 0,60 & $13,61 \mathrm{a}$ & $16,28 \mathrm{a}$ & $14,83 \mathrm{~b}$ & $15,46 \mathrm{~b}$ & $1815,5 \mathrm{a}$ \\
\hline & 0,80 & $13,60 \mathrm{a}$ & $14,93 \mathrm{a}$ & $18,50 \mathrm{a}$ & $16,15 \mathrm{a}$ & $1622,6 \mathrm{~b}$ \\
\hline \multirow{3}{*}{ DP } & 0,20 & $13,09 \mathrm{a}$ & $14,06 \mathrm{~b}$ & $14,89 \mathrm{~b}$ & $14,76 \mathrm{~b}$ & $2027,2 \mathrm{a}$ \\
\hline & 0,30 & $13,66 \mathrm{a}$ & $15,80 \mathrm{ab}$ & $16,56 \mathrm{ab}$ & $16,22 \mathrm{a}$ & $1614,3 \mathrm{~b}$ \\
\hline & 0,40 & $14,08 \mathrm{a}$ & $16,95 \mathrm{a}$ & $18,54 \mathrm{a}$ & $16,43 \mathrm{a}$ & $1515,6 \mathrm{~b}$ \\
\hline
\end{tabular}

Medias con las mismas letras no difieren significativamente, según la prueba de Tukey al 5 \% de probabilidad, comparación vertical. DH: distancia entre hileras; DP: distancia entre plantas. 
Tabla 5. Matriz de correlación y significancia estadística de rendimiento de semillas por hectárea (REND), altura de planta (AP), área foliar (AF), índice de área foliar (IAF), tasa de crecimiento del cultivo (TCC), longitud de la vaina (LV), número de vainas por planta (NVP) y peso de cien semillas (PCS) de frijol caupí cultivar Caupicor 50, a diferentes espaciamientos.planta (VP), peso de cien semillas (PCS) y rendimiento de semillas (REND) de fríjol caupí cultivar Caupicor 50, a diferentes espaciamientos.

\begin{tabular}{|c|c|c|c|c|c|c|c|}
\hline Variables & REND & AP & AF & IAF & TCC & LV & NVP \\
\hline AP & $-0,523^{* *}$ & & & & & & \\
\hline AF & $-0,536^{* *}$ & $0,506^{* *}$ & & & & & \\
\hline IAF & $0,480^{* *}$ & $-0,524^{* *}$ & $0,020^{\text {ns }}$ & & & & \\
\hline TCC & $0,306^{*}$ & $-0,489^{* *}$ & $-0,253^{\text {ns }}$ & $0,718^{* *}$ & & & \\
\hline LV & $-0,052^{\text {ns }}$ & $0,299^{\text {ns }}$ & $0,275^{\text {ns }}$ & $-0,224^{\text {ns }}$ & $-0,448^{* *}$ & & \\
\hline NVP & $-0,622^{* *}$ & $0,610^{* *}$ & $0,521^{* *}$ & $-0,375^{*}$ & $-0,345^{*}$ & $0,116^{\text {ns }}$ & \\
\hline PCS & $-0,354^{*}$ & $0,517^{* *}$ & $0,155^{\text {ns }}$ & $-0,665^{* *}$ & $-0,755^{* *}$ & $0,295^{\text {ns }}$ & $0,402^{*}$ \\
\hline
\end{tabular}

Significancia estadística de la correlación: ns: $\mathrm{p} \geq 0,05 ;{ }^{*} \mathrm{p}<0,05 ;{ }^{* *} \mathrm{p}<0,01$.

La LV aumentó en la medida en que la DP se hizo más amplio. El modelo cuadrático ajustado $\mathrm{Y}=-0,0071 \mathrm{X}^{2}+0,635 \mathrm{X}+3,4378$, $\mathrm{R} 2=0,79$, el cual, es similar al reportado por Soares de Melo et al. (2018), permite estimar un incremento lineal de $0,635 \mathrm{~cm}$ en la LV por cada $\mathrm{cm}$ que se aumente la DP y una desviación de la linealidad de $-0,0071 \mathrm{~cm}$ por cada $\mathrm{cm}$.

El NVP varió, según DH y DP, con mayores valores en los espaciamientos mayores, esto es en $80 \mathrm{~cm}$ entre hileras y $40 \mathrm{~cm}$ entre plantas, lo que equivale a la DP más baja, 31.250 plantas ha ${ }^{-1}$ (Tabla 4). Da Camara et al. (2018), también reportaron diferencias significativas en el NVP en la distancia entre hileras. La diferencia en el NVP entre la distancia entre plantas más alta, $40 \mathrm{~cm}$ y la más baja, $20 \mathrm{~cm}$, fue de 17,5\%. Esta diferencia para la distancia entre hileras fue de 19,8\%. NVP presentó correlación positiva con AP y AF y negativa, con IAF, TCC y REND (Tabla 5).

Por otro lado, Cardoso et al. (2018) reportaron un decrecimiento lineal del número de vainas por plantas al aumentar la población de plantas por hectárea, variando DH y DP, señalando que el número de vainas por planta máximo por $\mathrm{m}^{2}$ fue de 70,12 , con 19,6 plantas $/ \mathrm{m}^{2}$, en cultivares de hábito erecto; 65,6 vainas por $\mathrm{m}^{2}$ con 19,4 plantas $/ \mathrm{m}^{2}$, en cultivares semipostrados y 59,22 vainas por $\mathrm{m}^{2}$ con 15,6 plantas $/ \mathrm{m}^{2}$, en cultivares postrados

Para el NVP, se ajustó el modelo exponencial $\mathrm{Y}=9,469 \mathrm{e} 0,019 \mathrm{X}$, $\mathrm{R}^{2}=0,5532$, en el que el NVP se incrementa con una tasa de proporcionalidad es 0,019 , por cada $\mathrm{cm}$ de aumento de DP.

El PCS resultó 4,3\% menor en DH de $60 \mathrm{~cm}$ y 10,2 \% menor en la DP de $20 \mathrm{~cm}$ con respecto a la DP, de $40 \mathrm{~cm}$ (Tabla 4); en términos de densidad de plantas por hectárea, equivale a pasar de 31.250 a 83.333 plantas ha $a^{-1}$ y estuvo correlacionada positivamente con AP y NVP y, negativamente, con IAF, TCC y REND (Tabla 5). Resultados similares fueron encontrados por Kamara et al. (2018), quienes reportaron que el peso de las semillas se redujo en un $5 \mathrm{y}$ $9 \%$, cuando se pasó de 133.000 a 400.000 plantas ha ${ }^{-1}$, situación que, según estos autores, obedece a un mejor aprovechamiento de los nutrientes, agua, luz y distribución de los fotoasimilados bajo menores densidades de plantas, por unidad de superficie.

Para el PCS, se ajustó el modelo cuadrático $Y=-0,0086 \mathrm{X}^{2}+0,6219$ $X+5,4715, R^{2}=0,62$. Según este modelo, el PCS se incrementa en $0,6219 \mathrm{~g}$ por cada $\mathrm{cm}$ de aumento de DP, con un desvío de la linealidad de $-0,0086 \mathrm{~g}$, por cada $\mathrm{cm}$.

El REND aumentó con la disminución de DH y DP, es decir, conforme aumentó la densidad de plantas y estuvo correlacionado positivamente con el IAF y, negativamente, con NVP, PCS, AP y AF (Tabla 5). El aumento de la población de plantas $/ \mathrm{ha}^{-1}$ generó disminuciones en el número y tamaño de los órganos reproductivos, pero aumentó el IAF y el REND, en esta especie.

La correlación negativa del REND con NVP y PCS obedece a que con las mayores densidades de plantas se produce una competencia, principalmente por luz, cuyo efecto es de naturaleza individual y en un bajo porcentaje, por lo que las altas densidades de plantas son compensadas por la sumatoria de la producción de una mayor cantidad de plantas por unidad de superficie (Cardoso et al. 2018). Resultado similar fue reportado por De Sousa Monteiro et al. (2017), Kamara et al. (2018) y Da Camara et al. (2018), cuando incrementaron las densidades de población en estudios realizados con cultivares mejorados y criollos, tanto en Brasil como en Nigeria.

En el presente estudio, se obtuvo mayor REND con DP de $20 \mathrm{~cm}$, superior en $25,2 \%$ al obtenido con DP, de $40 \mathrm{~cm}$. Asimismo, con DH de $60 \mathrm{~cm}$, se obtuvo un REND 10,6\% mayor que con $80 \mathrm{~cm}$. Es evidente que, con la mayor densidad de plantas, 83.333 plantas $\mathrm{ha}^{-1}$, se alcanzó un mayor REND, con menor NVP y menor PCS, pero dentro de los rangos de comercialización de la semilla como grano, lo cual, representa, potencialmente, un ingreso mayor para el productor.

Mediante análisis de regresión, se ajustó el modelo cuadrático $\mathrm{Y}=$ 1,5708 X2-119,83 X + 3,795, R2=0,66, con el que se estima una disminución de 119,83 $\mathrm{kg}$ de REND, por cada cm de aumento de la 
distancia entre plantas, con un desvío del efecto lineal equivalente a $1,57 \mathrm{~kg}$.

Con DH de $60 \mathrm{~cm}$ y DP de $20 \mathrm{~cm}$, se logró el mayor rendimiento de semillas por unidad de superficie, debido al incremento IAF y la TCC, a pesar de la disminución del NVP y el PCS.

Conflicto de interés: El manuscrito fue preparado y revisado con la participación de todos los autores, quienes declaramos que no existe conflicto de interés que ponga en riesgo la validez de los resultados presentados.

\section{REFERENCIAS}

1. ALVES BASTOS, E.A.; DO NASCIMENTO, S.P.; DA SILVA, E.M.; FREIRE FILHO, F.F.; GOMIDE, R.L. 2011. Identification of cowpea genotypes for drought tolerance. Revista Ciência Agronômica (Brasil). 42(1):100-107.

https://doi.org/10.1590/S1806-66902011000100013

2. ALVES BASTOS, E.A.; MOREIRA RAMOS, H.M.; SOARES DE ANDRADE JÚNIOR, A.; NUNES DO NASCIMENTO, F.; CARDOSO, M.J. 2012. Parâmetros fisiológicos e produtividade de grãos verdes do feijãocaupi sob déficit hídrico. Water Resources and Irrigation Management (Brasil). 1(1):31-37.

3. APÁEZ BARRIOS, P.; ESCALANTE ESTRADA, J.A.S.; SOSA MONTES, E.; APÁEZ BARIOS, M.; RODRÍGUEZ GONZÁLEZ, M.T.; RAYA MONTAÑO, Y.A. 2016. Producción y calidad nutrimental de vaina del frijol chino, Vigna unguiculata (L.) Walp, en función de arreglo topológico y tipo de fertilización. Rev. FCA UNCUYO. (Argentina). 48(2):31-42.

4. ARAMÉNDIZ-TATIS, H.; CARDONA-AYALA, C.; JARMA, A.; COMBATT, E.; JARABA, J.; MERCADO, T.; ESPITIACAMACHO, M.; DE PAULA, C.; PASTRANA, Y.; HERNÁNDEZ, J. 2019. Manejo agronómico del fríjol caupí en el Caribe colombiano. Universidad de Córdoba. 22p.

5. BARRERA, J.L.; CARTAGENA, J.R.; NANCLARES, O. 2021. Influence of high density planting and spatial arrangement on growth and development in Musa AAA Simmonds. Rev. U.D.C.A Act. \& Div. Cient. (Colombia). 24(1):e1312. https://doi.org/10.31910/rudca.v24.n1.2021.1312

6. CARDOSO, M.J.; DE BRITO MELO, F; QUEIROZ RIBEIRO, V. 2018. Population density on cowpea cultivars with different growth habits in the matopiba region. Rev. Caatinga. (Brasil). 31(1):235-239.

http://dx.doi.org/10.1590/1983-21252018v31n127rc

7. COSTA JUNIOR, M.J.N.; ALVES BASTOS, E.; CARDOSO, M.J.; ANDRADE JUNIOR, A. 2017. Agronomic performance of the cowpea under different irrigation depths and row spacing. Rev. Ciência Agronômica. (Brasil). 48(5 Especial):774-782.

http://dx.doi.org/10.5935/1806-6690.20170090

8. DA CAMARA, F.T.; DUARTE MOTA, A.M.; DE ARAÚJO NICOLAU, F.E.; ALVES PINTO, A.; FRANCIELE DA SILVA, J.M. 2018. Produtividade de feijão caupi crioulo em função do espaçamento entre linhas e número de plantas por cova. Revista de Agricultura Neotropical. (Brasil). 5(2):19-24.

https://doi.org/10.32404/rean.v5i2.2282

9. DE CARVALHO BEZERRA, A.A.; ALVES FERNANDES TÁVORA, F.J.; FREIRE FILHO, F.R.; QUEIROZ RIBEIRO, V. 2009. Características de dossel e de rendimento em feijão-caupi ereto em diferentes densidades populacionais. Pesquisa Agropecuária Brasileira (Brasil). 44(10):1239-1245.

https://doi.org/10.1590/S0100-204X2009001000005

10. DE CARVALHO BEZERRA, A.A.; CABRAL DAS NEVES, A.; DE MOURA ROCHA, M.; RODRIGUES DE BRITO, L.C. 2017. Morpho-physiological and productive biometry in semi-erect cultivars of the cowpea under different plant population. Rev. Ciência Agronômica (Brasil). 48(4):625-630. http://dx.doi.org/10.5935/1806-6690.20170072

11. DE SOUSA MENDES, R.M.; ALVES FERNADES TÁVORA, F.J.; NUNES DE PINHO, J.L.; BOSCO PITOMBEIRA, J. 2005. Alterações na relação fonte-dreno em feijão-de corda submetido a diferentes densidades de plantas. Revista Ciência Agronômica (Brasil). 36(1):82-90.

12. DE SOUSA MONTEIRO, M.M.; ALVES BASTOS, E.; CARDOSO, M.J.; SOARES DE ANDRADE JÚNIOR, A.; QUEIROZ RIBEIRO, V. 2017. Effect of water regimes and plant densities on cowpea production. Pesq. Agropec. Trop. (Brasil). 47(4):432-439. https://doi.org/10.1590/1983-40632017v4747908

13. EL NAIM, A.M.; JABERELDAR, A.A. 2010. Effect of plant density and cultivar on growth and yield of cowpea (Vigna unguiculata L. Walp). Australian J. Basic and Applied Sciences. (Australia). 4(8):3148-3153.

14. GONÇALVES, I.S.; DA SILVA, R.R.; DE OLIVEIRA, G.M.; PINTO SANTIAGO, E.J.; ALVES DE OLIVEIRA, V.E. 2017. Características fisiológicas e componentes de produção de feijão caupi sob diferentes lâminas de irrigação. J. Environmental Analysis and Progress (Brasil). 2(3):320-329.

http://dx.doi.org/10.24221/jeap.2.3.2017.1456.320-329

15. HISSENE, H.M.; VADEZ, V.; CLAVIJO MICHELANGELI, J.; HALILOU, O.; NDOYE, I.; SOLTANI, A.; SINCLAIR, 
T. 2016. Quantifying Leaf Area Development Parameters for Cowpea [Vigna unguiculata (L.) Walpers]. Crop Science (USA). 56(6):3209-3217.

https://doi.org/10.2135/cropsci2016.02.0132

16. HUYNH, B.L.; CLOSE, T.J.; ROBERTS, P.A.; HU, Z.; WANAMAKER, S.; LUCAS, M.R.; CHIULELE, R.; CISSÉ, N.; ANTONIO, D.; HEARNE, S.; FATOKUN, C.; DIOP, N.N.; EHLERS, J.D. 2013. Gene pools and the genetic architecture of domesticated cowpea. The plant genome (USA). 6(3):1-8.

https://doi.org/10.3835/plantgenome2013.03.0005

17. INSTITUTO DE HIDROLOGÍA, METEOROLOGÍA Y ESTUDIOS AMBIENTALES, IDEAM. 2014. Tiempo y Clima. Promedios Climatológicos 1981-2010. Disponible desde Internet en

http://www.ideam.gov.co/web/tiempo-y-clima/clima (con acceso el 18/06/2020).

18. JAKUSKO, B.B.; ANASUNDA, U.I.; MUSTAPHA, A.B. 2013. Effect of inter-row spacing on some selected Cowpea (Vigna unguiculata (L) Walp) varieties in Yola, Adamawa State, Nigeria. IOSR J Agric Vet Sci. 2(1):30-35.

https://doi.org/10.9790/2380-0233035

19. JIMÉNEZ GALINDO, J.C.; ACOSTA GALLEGOS, J.A. 2013. Efecto de la densidad a simple y doble hilera en el rendimiento de frijol de temporal en Chihuahua, México. Rev. Mexicana de Ciencias Agrícolas (México). 4(3):393-407. http://dx.doi.org/10.29312/remexca.v4i3.1201

20. KAMARA, A.Y.; TOFA, A.I.; KYEI-BOAHEN, S.; SOLOMON, R.; AJEIGBE, H.A.; KAMAI, N. 2018. Effects of plant density on the performance of cowpea in Nigerian savannas. Experimental Agriculture (Inglaterra). 54(1):120-132.

https://doi.org/10.1017/S0014479716000715

21. KURUMA, R.W.; SHEUNDA, P.; KAHWAGA, C.M. 2019. Yield stability and farmer preference of cowpea (Vigna unguiculata) lines in semi-arid eastern Kenya. Afrika Focus (Bélgica). 32(2):65-82.

https://doi.org/10.21825/af.v32i2.15768
22. MAKOI, J.H.J.R.; CHIMPHANGO, S.B.M.; DAKORA, F.D. 2010. Photosynthesis, water-use efficiency and 813C of five cowpea genotypes grown in mixed culture and at different densities with sorghum. Photosynthetica. 48(1):143-155. http://dx.doi.org/10.1007/s11099-010-0019-2

23. PEDROZA-SANDOVAL, A.; YÁÑEZ-CHÁVEZ, L.G.; SÁNCHEZ-COHEN, I.; SAMANIEGO-GAXIOLA, J.A. 2015. Efecto del hidrogel y vermicomposta en la producción de maíz. Revista fitotecnia mexicana (México). 38(4):375-381.

https://doi.org/10.35196/rfm.2015.4.375

24. SOARES DE MELO, A.S.; FERREIRA DA SILVA, A.R.; FILGUEIRAS DUTRA, A.; FILGUEIRAS DUTRA, W; DA SILVA SÁ, F.V.; DE MOURA ROCHA, M. 2018. Crescimento e pigmentos cloroplastídicos de genótipos de feijão vigna sob déficit hídrico. Rev. Brasileira de Agricultura Irrigada. 12(3):2579-2591.

https://doi.org/10.7127/rbai.v12n300698

25. SOUZA LOPES, M.B.; DE OLIVEIRA TAVARES, T.C.; ALVES VELOSO, D.; DA SILVA, N.C.; RIBEIRO FIDELIS, R. 2017. Cowpea bean production under water stress using hydrogels. Pesq. Agropec. Trop. (Brasil). 47(1):87-92.

https://doi.org/10.1590/1983-40632016v4743398

26. SOUZA PEREIRA, J.S.; OLSZEVSKI, N.; CRUZ DA SILVA, J. 2018. Retenção de água e desenvolvimento do feijão caupi em função do uso de polímero hidrorretentor no solo. Revista Engenharia na Agricultura (Brasil). 26(6):582-591. https://doi.org/10.13083/reveng.v26i6.857

27. WUBETU, A. 2018. Effects of Intra and Inter-Row Spacing on Yield and Yield Components of Mung Bean (Vigna radiate L.). J. Biology, Agriculture and Healthcare (India). 8(12):1-9. 